\title{
Cuidados de enfermagem as vítimas de violências interpessoais na atenção básica
}

\author{
Nursing care for victims of interpersonal violence in basic attention \\ Cuidados de enfermería las víctimas de violencias interpersonales en la atención básica
}

\section{Amanda da Cunha Moreira ${ }^{1 *}$, Thiago Augusto Soares Monteiro da Silva ${ }^{2}$}

Como citar esse artigo. Moreira, $\mathrm{AC}$; da Silva, TASM. Cuidados de enfermagem as vítimas de violências interpessoais na atenção básica. Revista Pró-UniverSUS. 2019 Jan./Jun.; 10 (1): 42-46

\section{Resumo}

O estudo tem como objetivo descrever como os enfermeiros identificam situações de violência na Atenção Básica. O cenário escolhido foram 10 Estratégias de Saúde da Família em um município da região Centro Sul Fluminense, e para garantir o anonimato e sigilo as unidades serão identificadas por códigos alfanuméricos. Os participantes da pesquisa foram 20 profissionais de Enfermagem (auxiliar e técnico de enfermagem e Enfermeiros) que a atuam nas unidades. Foi realizada uma entrevista com um instrumento de perguntas e respostas abertas e fechadas. Após a coleta de dados procedeu-se a análise de conteúdo. A equipe de Enfermagem apresenta muita dificuldade em detectar a violência e as intervenções possíveis para cada caso, quando questionados sobre o que consideravam violência, todos tiveram respostas conclusivas sobre o que dizem ser violência. A equipe de enfermagem precisa receber, de profissionais especializados, uma capacitação para que possam saber detectar possíveis causas de violência e como agir nessas situações.

Palavras-chave: Enfermagem; Atenção Básica; Violência.

\begin{abstract}
The study aims to describe how nurses identify situations of violence in Primary Care. The scenario chosen was 10 Family Health Strategies in a municipality in the Centro Sul Fluminense region, and to ensure anonymity and confidentiality, the units will be identified by alphanumeric codes. The participants of the research were 20 Nursing professionals (auxiliary and nursing technician and Nurses) who work in the units. An interview with an open and closed question and answer instrument was conducted. After data collection, the content analysis was performed. The Nursing team presents a lot of difficulty in detecting violence and the possible interventions for each case, when asked about what they considered violence, all had conclusive answers about what they say is violence. The nursing team needs to receive, from specialized professionals, a training so that they can know to detect possible causes of violence and how to act in these situations.
\end{abstract}

Keywords: Basic Attention; Nursing; Violence.

\section{Resumen}

El estudio tiene como objetivo describir cómo los enfermeros identifican situaciones de violencia en la Atención Básica. El escenario escogido fue 10 Estrategias de Salud de la Familia en un municipio de la región Centro Sur Fluminense, y para garantizar el anonimato y sigilo las unidades serán identificadas por códigos alfanuméricos. Los participantes de la investigación fueron 20 profesionales de Enfermería (auxiliar y técnico de enfermería y Enfermeros) que la actúan en las unidades. Se realizó una entrevista con un instrumento de preguntas y respuestas abiertas y cerradas. Después de la recolección de datos se procedió a análisis de contenido. El equipo de enfermería presenta mucha dificultad en detectar la violencia y las intervenciones posibles para cada caso, cuando se les preguntó sobre lo que consideraban violencia, todos tuvieron respuestas concluyentes sobre lo que dicen ser violencia. El equipo de enfermería necesita recibir, de profesionales especializados, una capacitación para que puedan saber detectar posibles causas de violencia y cómo actuar en esas situaciones.

Palabras clave: Enfermería; Atención Básica; Violencia. 


\section{Introdução}

No Brasil, a violência é uma das principais causas de morbimortalidade, deixando de ser considerada um problema jurídico e social para ser incluído na área da saúde pública. ${ }^{1}$

Em face a esse cenário o enfermeiro na atenção básica acaba tendo um grande desafio, pois mantém freqüente contato com o cliente, porém em muitas situações não tem a visão para identificar que o mesmo pode estar sendo vítima de violência interpessoal ou doméstica, ou não sabe lidar com a situação.

$\mathrm{O}$ atendimento as vítimas de violências são constantes no cotidiano de cuidados de Enfermagem na Atenção Básica durante visitas domiciliares ou consultas na Estratégia de Saúde da Família (ESF), porém, a equipe de Enfermagem pode não deter a devida atenção durante o atendimento e não detectar sinais de vitimização e riscos que a vítima pode estar enfrentando ao se manter próxima do agressor.

$\mathrm{Na}$ Atenção básica, é onde deveria ser a porta de entrada dos pacientes. $\mathrm{O}$ estudo pode ser importante para o Enfermeiro gestor da unidade para o planejamento do cuidado às vítimas e o fluxo de atendimento ao mesmo. $\mathrm{O}$ estudo pode melhorar a assistência aos clientes que são vítimas de violência, e que vão até a ESF em busca de ajuda.

O presente estudo tem por objetivo descrever como os enfermeiros identificam situações de violência na Atenção Básica.

\section{Metodologia}

Trata-se de uma pesquisa descritiva, exploratória de abordagem qualitativa. $\mathrm{O}$ cenário escolhido para o desenvolvimento do estudo foram 10 Unidades Básicas de Saúde em um município da região Centro Sul Fluminense, e para garantir o anonimato e sigilo as Unidades foram identificadas por códigos alfanuméricos (E1, E2, E3 para enfermeiros e T1, T2, T3 para técnicos de Enfermagem). A escolha das mesmas foi feita conforme a conveniência de deslocamento e permanência do pesquisador para a coleta de dados.

Nessa perspectiva, solicitou-se a autorização, por escrito, a Secretaria de Saúde e ao setor de Atenção Básica do município, oportunidade em que se recebeu autorização para a coleta de dados nas Unidades Básicas de Saúde.

Os participantes da pesquisa compreenderam 20 profissionais da equipe de Enfermagem que realizam cuidados diretos e indiretos as vítimas de violências interpessoais na Unidade Básica de Saúde.

O profissional que se dispôs a participar da pesquisa recebeu um termo de Consentimento Livre e Esclarecido sendo informado sobre os objetivos da pesquisa assegurado os aspectos éticos da pesquisa com seres humanos conforme a Resolução 466/12. Garantindo o anonimato e também a possibilidade de se recusar a participar da pesquisa, bem como interromper sua participação em qualquer momento caso desejassem.

O presente estudo foi submetido á aprovação do Comitê de ética em Pesquisa (CEP) da Universidade de Vassouras, CAAE: 96137518.7.0000.5290, sob o número 2.874.134, oportunidade em que recebeu aprovação e liberação para início da pesquisa de campo.

Foram incluídos na pesquisa enfermeiros, técnicos de enfermagem e auxiliares de enfermagem de ambos os sexos com pelo menos 2 anos de atuação em atenção básica. Foram excluídos da pesquisa o profissional que estiver de férias ou licença no período da pesquisa.

A participação dos profissionais manteve o rigor ético, não promovendo nenhum tipo de complicações legais ou a sua saúde para os participantes e envolveu os riscos de desconforto e/ou constrangimento em responder alguma pergunta. Mas qualquer desconforto gerado pode ser amenizado em conversa com o pesquisador na retirada de dúvidas e/ou esclarecimentos.

Os benefícios do estudo consistem em pensar as possibilidades de cuidar das vítimas de violências interpessoais no cenário da Atenção Básica de Saúde.

$\mathrm{O}$ instrumento utilizado para coleta de dados foi um roteiro de entrevista composto de perguntas sociodemográficos, e perguntas abertas sobre: $\mathrm{O}$ que você entende como uma situação de violência? Você já atendeu na ESF vítimas de violência interpessoal? Como foi? Quando avalia um usuário, vítima de violência interpessoal através de quais sinais e sintomas você detecta a violência? O que você, como profissional, acha que pode ser feito para ajudar um cliente que foi vítima de violência? Você conhece a Enfermagem Forense? Ao realizar cuidados a uma vítima de violência interpessoal como você pode contribuir com a justiça?

Na segunda etapa foi realizada a coleta de dados durante o turno de funcionamento das Unidades Básicas de Saúde no período correspondente ao mês de outubro e início do mês de novembro do ano 2018 e os dados foram registrados no roteiro de entrevista.

A terceira etapa correspondeu a leitura do material e análise dos dados, produzido de acordo com a metodologia e à luz da Análise do Conteúdo.

Para realização da análise dos dados, inicialmente serão agrupamos as informações colhidas em concordância com os objetivos da pesquisa e roteiro de entrevista, será realizada uma leitura exaustiva dos dados com destaque através de cores e símbolos 
dos resultados evidenciando os que são convergentes e os que são divergentes, o que possibilitará gerar as categorias de análise.

\section{Resultados e Discussão}

\section{Perfil sociodemográficos da equipe de Enfermagem}

Quanto a faixa etária 55\% (11) apresentam de 30 a 39 anos, $30 \%$ (6) apresentam de 40 a 49 anos, $10 \%$ (2) apresentam de 20 a 29 anos e 5\% (1) de 50 a 59 anos. A média de idade encontrada foi de 37,7 anos variando de 27 a 53 anos.

Quanto a formação dos profissionais entrevistados $55 \%$ (11) são enfermeiros e $45 \%$ (9) são técnicos ou auxiliares de enfermagem. O que justifica maior quantidade de enfermeiros, é que no município da pesquisa, nas unidades da zona urbana, trabalha-se 2 enfermeiros por unidade.

Quanto ao sexo dos 20 profissionais entrevistados, apenas 5\% (1) é do sexo masculino, e 95\% (19) são do sexo feminino, o percentual maior de mulheres predomina. Nos dias atuais a Enfermagem permanece como profissão essencialmente feminina. ${ }^{2} \mathrm{~A}$ equipe de enfermagem é predominantemente feminina, sendo composta por $84,6 \%$ de mulheres. ${ }^{3}$

Quanto ao tempo de graduação $50 \%$ (10) dos entrevistados tem de 6 a 10 anos, $40 \%$ (8) tem de 11 a 20 anos e $10 \%$ (2) tem de 21 a 30 anos. A média encontrada de tempo de graduação foi de 11,75 anos variando de 6 anos a 22 anos.

Quanto ao tempo de trabalho prestado na atenção básica $50 \%$ (10) trabalham entre 2 a 5 anos, 30\% (6) trabalham de 6 a 10 anos e $20 \%$ (4) trabalham entre 11 a20 anos. Uma média de atuação de 7,15 anos de atuação. Machado et al (2016) diz que "quanto maior o tempo de atuação, possibilita o vínculo do cuidado".

\section{Conceito de violência apresentado pela equipe de Enfermagem}

A equipe de enfermagem entrevistada entende por violência qualquer tipo de agressão ou abuso de poder ao indivíduo, família, comunidade sendo ela física, psicológica, moral, patrimonial, verbal, social e sexual. $\mathrm{O}$ início pode ser sútil e piorar ao longo do tempo. A situação muitas vezes vem oculta, disfarçada, podendo ser imperceptível, levando a causar danos irreversíveis como acidentes, danos psicológicos ou a morte.

Qualquer ato ou fala que possa gerar constrangimento ou lesionar física e emocionalmente alguém. (E7) Qualquer ação física, verbal ou usodo poder que possa resultar em algum dano físico, emocional, psicológico a outra pessoa. (E8) Toda equalquer atitude que uma

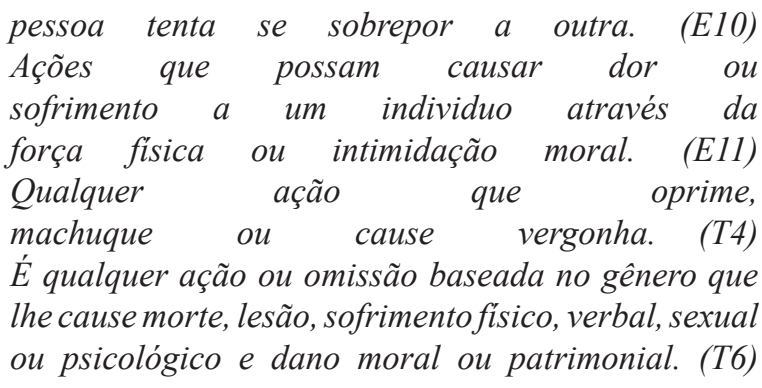

A violência é definida como uso intencional da força física ou do poder contra si próprio, contra outra pessoa, ou uma comunidade ou grupo, que resulte em lesão morte, dano psicológico, deficiência de desenvolvimento ou privação. ${ }^{4} \mathrm{~A}$ violência pode ser evitada, e suas consequências, reduzidas. ${ }^{5}$

Observa se que os profissionais da enfermagem em nenhum momento das entrevistas citaram como forma de violência a autodirigida, o que nos leva a acreditar que os profissionais podem ter dificuldades de identifica-la e consequentemente as vítimas podem ficar na "invisibilidade", deixando de serem notificados os casos. Os profissionais podem não identificar tal situação como um problema de saúde.

\section{Cuidados de Enfermagem às vítimas de violência na Atenção Básica}

Quando abordados sobre se já haviam atendidos vítimas de violência na unidade, $50 \%$ disseram já ter atendido, e $50 \%$ disseram nunca ter atendido. E quando questionados de como foi o atendimento as vítimas eles disseram que é sempre uma situação difícil e constrangedora, que acabam tendo conhecimento dos casos através de relato dos Agentes Comunitários de Saúde (ACS) e que na maioria das vezes eles realizam o acolhimento, que na visão deles é ouvir, dar apoio, orientar e aconselhar. Em alguns casos encaminhamento ao hospital e um entrevistado disse notificar.

$O$ paciente (cliente) tem dificuldades em relatar o que houve. Basicamente acabamos tomando ciência através do relato do ACS na maioria dos casos. (E1) A paciente encontrava-se vulnerável, triste. Fizemos orientações e aconselhamento. (E7) Sempre é uma situação dificil, constrangedora. (E10) Dentrodofluxogramaestabelecido, nãoseibemaocerto, sei que faz notificação, o núcleo de DST atende, tem os testes rápidos, em caso de menor (idade) o conselho tutelartem que seracionado. Profilaxia e gestação. (E6) Não violência física. Conversei, deixei a pessoa colocar para fora. Nada além de ouvir e dar apoio. (T1) Em alguns casos o agressor (a) está na cola do agredido dificultando o diálogo ou abordagem. (T5)

O acolhimento na Atenção Básica em saúde: relações de reciprocidade entre trabalhadores e usuários, que é uma ferramenta que possibilita a humanização do cuidado; ampliar o acesso da população aos serviços de 
saúde e assegurar a resolução dos problemas. ${ }^{6}$

Foi possível perceber com as entrevistas, que grande parte da equipe de enfermagem ao atender uma vitima de violência, falam sobre o acolhimento dessas vitimas, porém a maioria não fala em notificação, o que seria uma atitude muito importante desses profissionais para que assim tenha uma ideia da magnitude do problema e que se possa intervir com políticas de saúde publicas mais intensas.

Quantos aos sinais que a equipe de enfermagem consegue detectar uma situação de violência disseram sinais físicos, verbalização, mudanças no comportamento da vítima, recusa ou vergonha ao falar sobre o assunto, a pessoa fica mais chorosa, desvio do olhar, isolamento, baixa autoestima, depressiva e medo.

Introspecção, irritabilidade,
mudança de Através da fala, dos depoimentos, da atitude. (E 3) Geralmente a pessoa aparenta-se entristecida, desmotivada e por vezes recusa a falar do assunto. (E7) Geralmente são pessoas que não te olham nos olhos, demonstram nervosismo, pela experiência, observo que ocultam situações. Algumas vêm acompanhadas do agressor (E10) É possivel identificar a violência pelo jeito que o paciente se apresenta. Se o mesmo se encontra nervoso, ou contradiz. (T7) Muitos sentem vergonha e se intimidam diante da situação, quando chamados para um diálogo manifestam choro, insatisfação e medo. (T9)

Identificar a violência depende da mobilização de recursos internos, sensibilidade, habilidade e disponibilidade para ouvir o outro, além do intenso investimento em capacitação profissional. ${ }^{7}$

Identificar uma vítima de violência não é algo tão simples quanto parece, e uma das dificuldades que pode ser colocado pela equipe de enfermagem é a rotina e a falta de tempo. E é nesse ponto que a equipe precisa ter a sensibilidade e disponibilizar tempo para a escuta desses pacientes e assim poder ajudar ou até mesmo intervir de alguma forma.

Quando perguntou-se sobre como a equipe ajuda uma vítima de violência foram citados: apoio, encaminhamento, escutar, acolher, ajudar a denunciar, empatia, notificar, aconselhamento, orientar e cuidados físicos se necessário.

Oferecer escuta, apoio emocional, acolher, ajudar a diminuir os agravos físicos quando for o caso. (E2) Dependendo do tipo de agressão encaminhar para os conselhos, notificações, delegacias. (E5) Primeiramente acolher o cliente, escutar suas queixas, medos anseios e encaminhálo a especialidade mais adequada. (E11) Acho que deve ter um acompanhamento psicológico e também de apoio da familia e amigos. (T3)
Penso também em educar o agressor, ressocializar, cuidar ou tratar desta pessoa que agride. (T5) Orientar, conversar, ouvir e encaminhar aos seus respectivos órgãos para dar direção e cuidados, órgãos responsáveis. Temos que dar um bom acolhimento. (T6) Orientar o paciente que ás vezes a violência não necessariamente precisa ser física. (T7)

Com base nas discussões dos sujeitos, propõe se um fluxo para o atendimento as vitima de violência na Atenção Básica de Saúde.

$\mathrm{Na}$ visita domiciliar, na consulta de demanda espontânea ou na consulta de rotina na ESF identificouse uma vitima de violência. Após essa detecção faz se o acolhimento dessa vítima em uma sala reservada onde a vitima deve ser sentir a vontade. Logo após a escuta realizar a notificação, que deverá ser feita em duas vias, onde uma deve ser enviada ao setor de Epidemiologia para ser averiguada e a outra deve ser anexada ao prontuário da vítima ou á uma pasta própria de notificações.

Durante o acolhimento avaliar a necessidade do paciente quanto à consulta médica, caso não possa acontecer no momento ou não seja necessário avaliar as lesões. Se forem lesões graves, tais como baleado, esfaqueado ou com sangramento intenso acionar imediatamente o Serviço Móvel de Urgência (SAMU), se não apresentarem lesões graves deverá ter condutas diferenciadas para cada tipo de paciente.

No caso de criança ou adolescente, acionar conselho tutelar e a polícia. Em caso de adulto (mulher, gestante ou homem) aconselhamento, pois nesse caso somente a pessoa que foi agredida pode ter a iniciativa de denunciar. Em caso de violência sexual encaminhar ao setor de DST/AIDS para realização de medicamentos contraceptivos e/ou antirretroviral. Em caso de idoso o conselho do idoso deve ser acionado junto da polícia.

Uma das dificuldades que os profissionais podem ter em realizar a notificação ou a acionar os órgãos competentes é o medo dos perpetradores, mas é importante ressaltar que as denuncias devem ser confidenciais.

\section{Conclusão}

Pôde-se identificar que as equipes de enfermagem entrevistadas, atuantes na Atenção Básica do município de Vassouras, têm muita dificuldade em detectar a violência e as intervenções possíveis para cada caso.

Quando questionados sobre o que consideravam violência, todos tiveram respostas conclusivas sobre o que dizem ser violência. Porém quando questionados sobre as possíveis intervenções da equipe a uma vítima de violência, nenhum deles soube dar uma resposta concreta. Grande parte, diz em acolhimento, porém falam em encaminhar os casos a outros profissionais. Nenhum 
dos entrevistados citou como forma de violência, aquela que é gerada contra a si próprio, o que mais uma vez mostra que as equipes estão despreparadas para detectar sinais de violência.

A ficha de notificação do SINAN (Sistema de Informação de Agravos de Notificação), que pode ser preenchida em qualquer unidade de saúde, seja ela na atenção hospitalar ou atenção primária, em momento nenhum foi citada por nenhum dos entrevistados. Lembrando, que essa ficha não precisa ser preenchida apenas em casos confirmados de violência, mas também em casos suspeitos.

Chegou-se à conclusão de que a equipe de enfermagem precisa receber de profissionais especializados, uma capacitação para que possam saber detectar possíveis causas de violência e como agir nessas situações.

\section{Referências}

1. Oliveira SM, Fatha LCP, Rosa VL, Ferreira CD, Gomes GC, Xavier DM. Notificação de violência contra crianças e adolescentes: atuação de enfermeiros de unidades básicas. Rev. Enferm. UERJ, Rio de Janeiro, 2013 dez.; 21(1, n. esp):594-9.

2. Vall J, Pereira LF, Friesen TT. O perfil do acadêmico de enfermagem em uma faculdade privada da cidade de Curitiba. Cadernos da Escola de Saúde, 2017;1(2):1-10.

3. Machado Mh (Coord.), Aguiar Wf, Lacerda Wf,Oliveira E, Lemosw, Wermelinger M, Et. Al. Relatório final da Pesquisa Perfil da Enfermagem no Brasil (Convênio: FIOCRUZ/COFEN). Rio de Janeiro: 28 volumes, NERHUS-DAPS-Ensp/Fiocruz e Cofen; 2015.

4. OMS, Organização Mundial da saúde. Relatório Mundial Sobre Violência e Saúde. Genebra 2002; [Periódico disponível na Internet] Acesso em 15/11/2018. Disponível em: https://www.opas.org.br/wp-content/ uploads/2015/09/relatorio-mundial-violencia-saude.pd

5. Dahberg LL, Krug EG. Violência: Um problema de saúde pública Ciência \& Saúde Coletiva, 2007;11(Sup): 1163-78.

6. Lopes AS, Vilar RLA, Melo RHV, França RCS. O acolhimento na Atenção Básica em saúde: relações de reciprocidade entre trabalhadores e usuários. Saúde em Debate 2015 jan-mar; 39(104):114-23.

7. Baraldi ACP, Almeida AMD, Perdoná GC, Vieira EM. Violência contra a mulher na rede de atenção básica: o que os enfermeiros sabem sobre o problema? Revista Brasileira de Saúde Materno Infantil, 2012;12(3):30718 